\title{
Embarazo molar de 25 semanas con feto vivo. Reporte de autopsia
}

\author{
Cristina Mariela Flórez Flórez ${ }^{a}$, Diana Katherine Sandoval Martínez ${ }^{1}$, Olga Mercedes \\ Álvarez Ojeda ${ }^{1}$, Ernesto García Ayala. ${ }^{1}$ \\ ${ }^{1}$ Departamento de Patología, Grupo de Patología Estructural, Funcional y Clínica de la Universidad Industrial de \\ Santander (PAT-UIS). Escuela de Medicina, Facultad de Salud, Universidad Industrial de Santander. Bucaramanga, \\ Colombia.
}

a Alumna XII nivel de Medicina, Universidad Industrial de Santander. Bucaramanga, Colombia.

\section{RESUMEN}

Antecedentes: La mola hidatidiforme $(\mathrm{MH})$ se caracteriza por la degeneración hidrópica de las vellosidades coriales, hiperplasia del trofoblasto y se clasifica en completa (MC) y parcial (MP), y difieren en su cariotipo, histopatología, clínica y riesgo de malignidad. Constituye el 1\% de las pérdidas fetales en menores de 17 semanas. El objetivo de esta comunicación es exponer un caso de MP con feto de 25 semanas, al cual se le realizó autopsia en el 2014. Caso clínico: Primigesta de 21 años con pobre control prenatal y embarazo de 25 semanas con diagnóstico de preeclampsia y óbito fetal de sexo masculino de $615 \mathrm{~g}$, al que se le solicitó autopsia demostrándose malformaciones múltiples con agenesia del cuerpo calloso, hidrocefalia supratentorial, hipoplasia cerebelosa, meningocele sacro, micropene, hipospadias y retardo del crecimiento intrauterino, la placenta de $750 \mathrm{~g}$ demostró hallazgos de enfermedad trofoblástica gestacional de tipo MP. Discusión: La MP presenta vellosidades coriónicas con hiperplasia trofoblástica y feto que tiende a fallecer en una temprana edad gestacional. El feto puede presentar anomalías congénitas secundarias a la aneuploidia y la distinción se basa en histopatología de la placenta, en casos difíciles se emplea citogenética o citometría de flujo. En el presente caso se encontraron características placentarias (degeneración hidrópica vellositaria, inclusiones e hiperplasia trofoblástica) y las malformaciones fetales relacionadas. Conclusión: El estudio histopatológico de la placenta y del producto de la gestación permiten el diagnóstico definitivo para poder determinar el seguimiento de la paciente y disminuir así las complicaciones.

\section{PALABRAS CLAVE: Mola hidatidiforme parcial, aneuploidía, enfermedad trofoblástica gestacional}

\section{SUMMARY}

Background: Hydatidiform moles (HM) are characterized by hydropic degeneration of chorionic villi, hyperplasia of the trophoblast and is classified in complete (CM) and partial (PM), and they are different in their karyotype, histopathology, clinical and risk of malignancy. It constitutes $1 \%$ of abortion in under 17 weeks. The purpose of this communication is to present a case of PM with 25-week fetus, which fetal autopsy in 2014. Clinical case: Primigravid of 21 years old, with a pregnancy of 25 weeks diagnosed with preeclampsia and stillbirth of $615 \mathrm{~g}$, autopsy demonstrating multiple malformations with agenesis of corpus callosum, hydrocephalus, hypoplasia of the cerebellum, myeloschisis in the sacral region, micropenis, hypospadias and intrauterine growth retardation, the placenta $(750 \mathrm{~g})$ has findings of gestational trophoblastic disease 
type PM. Discussion: The PM has villous tissue with trophoblastic proliferation and fetus tends to die at an early gestational age. The fetus has congenital abnormalities because the aneuploidy, diagnoses is based on histopathology of the placenta, in difficult cases cytogenetic or flow cytometry is used. In this case, placental characteristics (hydropic villus, trophoblastic proliferation and "pseudoinclusions") and related fetal malformations were found. Conclusion: The histopathological study of the placenta and the fetus allow a definitive diagnosis to determine the monitoring of the patient and thus reduce complications.

\section{KEY WORDS: Partial hydatiform mole, aneuploidy, gestational trophoblast disease}

\section{INTRODUCCIÓN}

La mola hidatidiforme $(\mathrm{MH})$ hace parte de un grupo catalogado como enfermedad trofoblástica gestacional (ETG), y se caracteriza por la degeneración hidrópica de las vellosidades coriales con hiperplasia del trofoblasto y regularmente la ausencia del feto $(1,2)$. El embarazo molar se ha clasificado en dos grupos: la mola completa y la mola parcial, las cuales difieren en su cariotipo, histopatología, presentación clínica y riesgo de malignidad (1-5). La mola completa o clásica no posee feto o embrión, y generalmente su cariotipo es 46,XX o $46, X Y$, todo el material genético proviene del padre, por la fertilización de un solo espermatozoide de un "huevo vacío" con subsecuente duplicación del material genético y menos frecuentemente por dispérmica (fertilización de un huevo vacío por 2 espermatozoides con fusión de los dos pronúcleos masculinos). Mientras que la mola parcial o incompleta son usualmente triploides (dos set de genes paternos y uno materno), pueden asociarse a la presencia de feto, embrión o saco gestacional y se desarrollan por la fertilización dispérmica de un óvulo $(1,6-9)$

El objetivo de esta comunicación es exponer un caso de mola parcial con feto de 25 semanas de edad gestacional al cual se le realizó autopsia en el Hospital Universitario de Santander/Departamento de Patología de la Universidad Industrial de Santander en el 2014, y hacer una revisión de la literatura de esta entidad.

\section{Caso clínico}

Mujer de 21 años primigesta, con embarazo de 25 semanas, con diagnóstico de preeclampsia se- vera y pobre control prenatal, en quien se atendió parto vaginal en podálica con ausencia de actividad cardiaca y pulsaciones del cordón, se envió cuerpo del óbito y la placenta para estudio para autopsia médico-científica. Feto de sexo masculino con retardo del crecimiento intrauterino, con peso de 615 gramos, talla $30,5 \mathrm{~cm}$, longitud corona a cóccix de $19 \mathrm{~cm}$, circunferencia craneana sobre el plano occipito-frontal de $21 \mathrm{~cm}$, perímetro torácico de $19 \mathrm{~cm}$ y perímetro abdominal de $15 \mathrm{~cm}$, a nivel craneano se identificó baja implantación de los pabellones auriculares, sin defectos de la línea media facial ni a nivel de la pared abdominal, a nivel dorsal se identificó meningocele sacro, y micropene con hipospadias (Figura 1). Al examen interno se reconoció malformaciones múltiples con afección del sistema nervioso central (agenesia del cuerpo calloso, hidrocefalia supratentorial, hipoplasia cerebelosa y meningocele sacro).

El disco placentario era irregular de $750 \mathrm{~g}$ de $20 \times 15 \times 4 \mathrm{~cm}$, muestra en la cara fetal membranas opacas sin lesiones y cordón velamentoso en el que se observó quiste amniótico de $4 \mathrm{~cm}$ de diámetro promedio el cual no muestra compromiso de las estructuras vasculares. La cara materna exhibe vellosidades hidrópicas de aspecto vesicular mezcladas con otras pequeñas de apariencia usual. Histológicamente las vellosidades coriónicas presentan degeneración hidrópica y cavitaciones (cisternas) con hiperplasia del trofoblasto y presencia de inclusiones (Figura 2).

Se consideró que la muerte fetal ocurrió in útero en las 6 horas previas al parto por hipoxia extraplacentaria de causa materna (trastorno hipertensivo asociado al embarazo) e insuficiencia útero-placentaria con hallazgos de enfermedad trofoblástica gestacional de tipo mola parcial. 

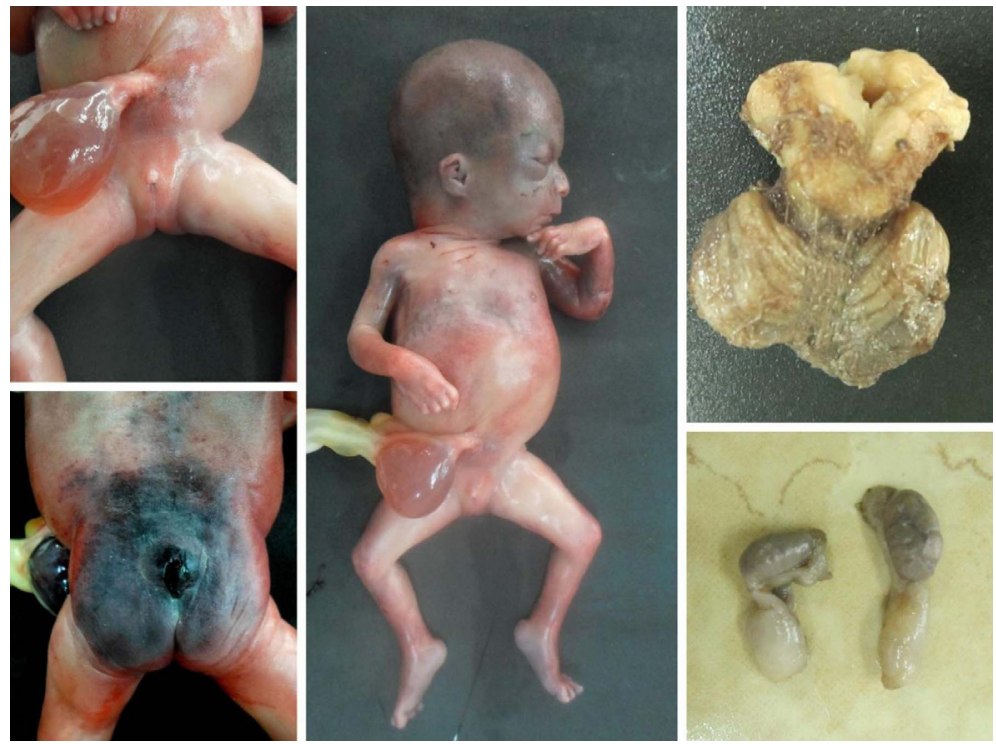

Figura 1. Feto 25 semanas que al examen macroscópico mostró baja implantación de los pabellones auriculares (imagen central) y quiste amniótico a nivel del cordón umbilical con micropene e hipospadia y bolsas escrotales casi imperceptibles (superior izquierda). En la región sacra hiperpigmentación cutánea con compromiso glúteo (inferior izquierda), identificando defecto del raquis de $0,7 \times 1 \mathrm{~cm}$ con protrusión de las meninges (meningocele sacro). Hipoplasia del cerebelo (superior derecha), y evidencia en cavidad abdominal de gónadas masculinas (inferior derecha).

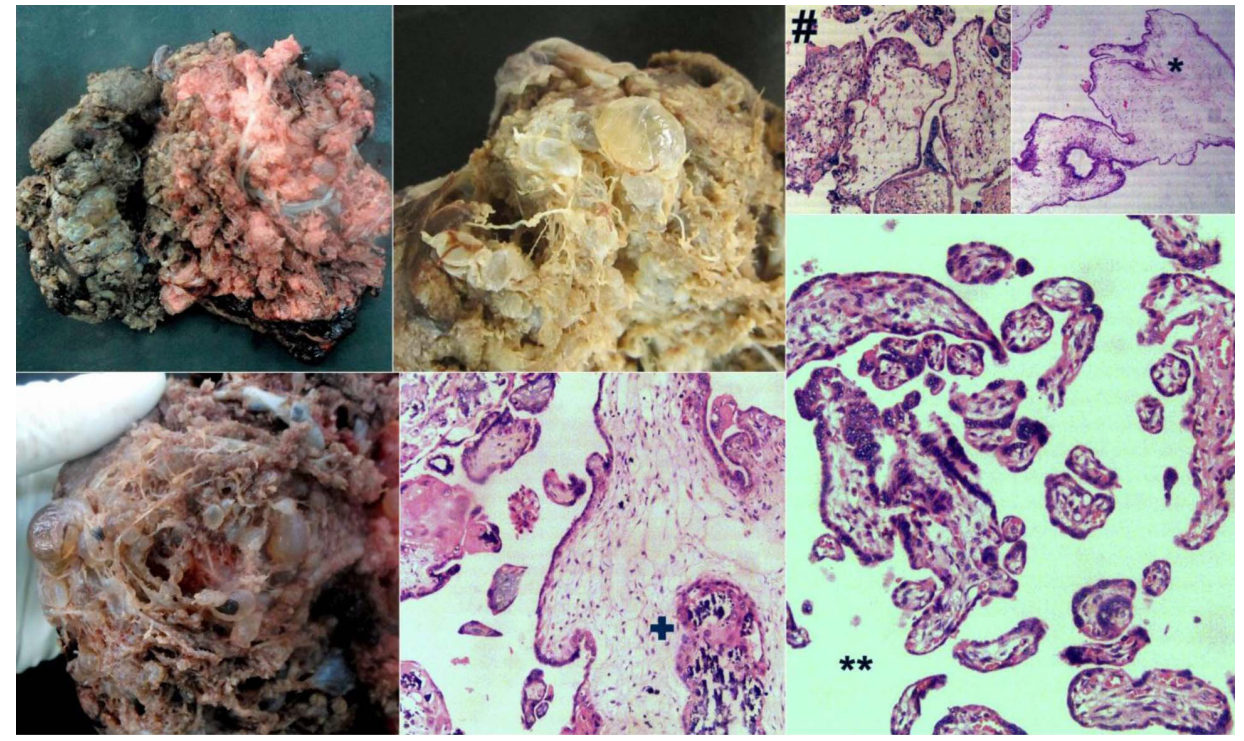

Figura 2. La imagen macroscópica muestra placenta con vellosidades hidrópicas de aspecto vesicular mezcladas con otras pequeñas de apariencia usual. Los cortes histológicos muestran vellosidades coriónicas con tamaños variables, algunas agrandadas con degeneración hidrópica y cisternas $\left(^{*}\right)$, otras con bordes festoneados con apariencia geográfica (\#), e hiperplasia del trofoblasto con presencia de inclusiones en algunas de estas (+), y en otras áreas exhiben fibrosis estromal son de pequeño tamaño con calcificaciones $\left({ }^{* *}\right)(\mathrm{H} \& \mathrm{E})$. 


\section{DISCUSIÓN}

En 1976 se clasificó la mola hidatidiforme en completa o parcial de acuerdo a las características morfológicas y citogenéticas $(1,10)$. La mola completa se caracteriza por una rápida progresión, una inflamación hidrópica de las vellosidades coriónicas e hiperplasia trofoblástica, ausencia de embrión o feto, y un cariotipo diploide de origen paterno (46,XX o 46,XY), y está asociado a enfermedad trofoblástica maligna; sus síntomas característicos son el sangrado vaginal irregular, aumento tamaño del útero, quistes ováricos, elevación marcada de la gonadotropina corionica humana ( $\beta-H C G)(2,4,5)$. La mola parcial presenta vellosidades coriónicas de diferentes tamaños, con progresión lenta y focal de la vellosidad que por contener vasos sanguíneos presentan menor cambio hidrópico, observándose hiperplasia trofoblástica $(1,2,4,8)$. También se pueden reconocer estructuras de embrión o feto que tiende a fallecer en una temprana edad gestacional por la presencia de complicaciones maternas y fetales, entre las que se destaca el desarrollo de trastorno hipertensivo asociado al embarazo de aparición temprana, que presenta rangos de severidad y puede observarse hasta en el $40 \%$ de los casos, si la gestación alcanza el segundo trimestre $(7,10)$. Cuando el feto se identifica hay retardo en el crecimiento intrauterino y anomalías congénitas asociadas a triploidia $(5,8)$.

Se estima que la mola parcial tiene una frecuencia de 1:700 de los embarazos con resultado adverso, pero los datos no son exactos porque en la mayoría de los casos no se diagnóstica y transcurren como abortos espontáneos del primer trimestre, se piensa que representan al $1 \%$ de las pérdidas fetales, entre las semanas 7 y 17, y los casos de nacidos vivos ocurren con una frecuencia de 1/100.000, por lo general los casos que sobreviven al término son mosaicos $(6,8,11,12)$. Existen dos formas en las que se puede presentar feto asociado a la presencia de mola hidatidiforme: huevo triploide dispérmico con mola parcial y feto, donde por lo general el feto está malformado y no es viable, o gemelar verdadero donde existe un embarazo normal asociado a una mola completa $(1,9)$.

Las placentas con mola parcial por lo general presentan la mayoría de las características patológicas y clínicas de la mola completa pero en forma atenuada $(4,13)$. La distinción se basa en el examen histopatológico y en casos difíciles se emplea la citogenética, citometría de flujo, o inmunohistoquímica (11). Las características histopatológicas empleadas para el diagnóstico de la mola parcial son: 1) Hiperplasia del sincitiotrofoblasto circunferencial o multifocal, 2) Edema focal de las vellosidades con cavitación, 3) Vellosidades con bordes irregulares, 4) Inclusiones trofoblásticas dentro del estroma vellositario $(8,9,14)$. Adicionalmente presentan fragmentos de tejido embrionario/fetal como vasos sanguíneos con glóbulos rojos, amnios, placa coriónicas, cordón umbilical $(1,10)$. Cuando los tejidos fetales son muy evidentes se pueden observar características de malformaciones (8).

Los fetos son pequeños para su edad gestacional con un crecimiento disarmónico, que pueden presentar malformaciones de sistema nervioso central como defectos de tubo neural (encefalocele, espina bífida), holoprosencefalia, agenesia de bulbos y tractos olfatorios (arrienencefalia), hipoplasia del cerebelo, agenesia del cuerpo calloso con alteraciones faciales como microftalmia, micrognatia, aplanamiento del puente nasal, hendidura labiopalatina, baja implantación de los pabellones auriculares, cuello corto, en los fetos masculinos se puede observar hipospadias, criptorquidia y micropene, y se ha descrito alteraciones internas como atresia intestinal, hernia diafragmática o inguinal, anomalías biliares, cardiopatía congénita y riñón poliquistico (9).

La presencia de mola parcial con feto vivo es inusual por la variedad de complicaciones clínicas materno-fetales que pueden presentarse, entre otras asociadas a la proliferación trofoblástica como son el desarrollo de preeclampsia temprana, hemorragias del primer trimestre, hipertiroidismo materno entre otros $(1,6,10)$. El seguimiento postparto va encaminado a detectar formas tempranas de enfermedad trofoblástica persistente y así evitar complicaciones ulteriores, siendo la medición sérica de los valores de la $\beta-H C G$ un marcador sensible, dado que esta es producida por el trofoblasto hiperplásico (7). En el presente caso se encontraron características placentarias que demostraron la degeneración hidrópica vellositaria asociado a la hiperplasia del trofoblasto, la presencia de inclusiones trofoblásticas y las malformaciones fetales relacionadas. En los controles clínicos ulteriores la paciente tuvo negativización de los controles séricos de $\beta$-HCG y los controles ecográficos no mostraron enfermedad trofoblástica persistente. El hallazgo de trastorno hipertensivo asociado al embarazo de desarrollo temprano puede hacer sospechar la presencia de $\mathrm{MH}$.

\section{CONCLUSIÓN}

El antecedente de $\mathrm{MH}$ constituye un factor de riesgo importante por la posibilidad de recurrencia o de presentar mola invasora o desarrollar coriocarcinoma, el adecuado estudio histopatológico de la placenta y los productos de la gestación permiten el diagnóstico definitivo para poder determinar el seguimiento de la paciente y disminuir así las posibles complicaciones. 


\section{REFERENCIAS}

1. Juárez Azpilcueta A, Islas Domínguez L, Durán Padilla MA. Mola hidatidiforme parcial con feto vivo del segundo trimestre. Rev Chil Obstet Ginecol 2010;75(2):137-9.

2. Dhingra KK, Gupta P, Saroha V, Akhila L, Khurana N. Partial hydatidiform mole with a full-term infant. Indian J Pathol Microbiol 2009;52:590-1.

3. Rice LW, Lage JM, Berkowitz RS, Goldstein DP, Bernstein MR. Repetitive complete and partial hydatidiform mole. Obstet Gynecol 1989;74(2):217-9.

4. Berkowitz RS, Goldstein DP, Bernstein MR. Natural history of partial molar pregnancy. Obstet Gynecol 1985;66(5):677-81.

5. Wood SJ, Sephton V, Searle T, Troup S, Kingsland Ch. Case of partial hydatidiform mole after intracytoplasmic sperm injection and assisted zona hatching. BJOG 2002;109:964-6.

6. Garc M, Bautista E. Caso clínico Embarazo molar parcial: reporte de un caso y revisión de la bibliografía. Ginecol Obstet Mex 2011;79(7):432-5

7. López Baños L, Oliva Rodríguez JA, Pérez Trujillo J, Hernández Pérez EP, Hernández Pérez T.
Mola hidatiforme parcial. Rev Cuba Obstet Ginecol 2012;38(3):431-7.

8. Gutiérrez C. Aproximación al diagnóstico de las anomalías del desarrollo. En: Gutierrez C. Temas de patología perinatal y pediátrica. Montevideo, Uruguay. BiblioMédica; pp247-50.

9. Baergen RN. Hydatidiform Moles. Chapter 23. Manual of Pathology of the Human Placenta. Second Edition. New York. Springer. pp 427-46.

10. Juárez AA, Durán PM, Islas DL, Martínez MS. Mola parcial con feto vivo, análisis clínico, morfológico y genético. Rev Mex Pediatr 2009;76(5):212-4.

11. Genest DR. Partial hydatidiform mole: clinicopathological features, differential diagnosis, ploidy and molecular studies, and gold standards for diagnosis. Int J Gynecol Pathol. 2001;20(4):315-22.

12. Goldstein DP, Horowitz NS. Hydatidiform mole: Epidemiology, clinical features, and diagnosis. Up to Date 2015:1-21.

13. Szulman AE, Surti U. The clinicopathologic profile of the partial mole. Obstet Gynecol 1982;59(5):597-602.

14. Howat A, Beek S, Fox H, Harris S, Hill A, Nicholson C, Williams RA. Can histopathologists reliably diagnose molar pregnancy? J Clin Pathol 1993;46(7):599-602. 